\title{
A Magnetostrictive Force and Vibration Mode Analysis of 3 kW BLDC Motor by a Magneto-Mechanical Coupling Formulation
}

\author{
Pan Seok Shin ${ }^{\dagger}$ and Hee Jun Cheung*
}

\begin{abstract}
This paper proposes a method to calculate magnetostrictive forces, displacement, and vibration modes of a large-scale Brushless DC(BLDC) motor by using a magneto-mechanically strong coupling formulation. The force is calculated using the energy method with magnetostrictive stress tensor. The mechanical vibration modes are also analyzed by using the principle of Hamilton and the calculated magneto-elastic forces acting on the surfaces of the stator. To verify the algorithm, $3 \mathrm{MW}$ BLDC motor is simulated, and the forces, displacements, and vibration modes are calculated. The result shows that the mechanically stressed core has more deformation or displacements than those of the normal condition.
\end{abstract}

Keywords: Electromagnetic force, Electrostriction, Magneto-mechanical coupling formulation, Motor vibration, Magnetostrictive force, BLDC motor, Vibration modes of stator

\section{Introduction}

The electromagnetic forces and displacement analysis of large-scale motor is very important for the design of the mechanical structure of motor as well as for the vibration of its system. In rotating electric machines, a magnetoelastic coupling phenomenon exists, which causes considerable changes in the mode and velocity of the vibrations of the large stator. Due to the existence of the coupling phenomenon, the conventional method of calculating the vibrations of electrical machines is less accurate compared with using coupled formulation. This paper presents a method to analyze the magnetostrictive force and vibration modes of a large-scale motor by using a magneto-mechanically strong coupling formulation. Since the electromagnetic and the elastic fields has an effect on each other, such coupling analysis is important and necessary for electric machines [1]-[3].

In general, the methods of analyzing the force acting on a ferromagnetic material with magnetostriction can be divided into two kinds: the weak and the strong coupling [4]. The weak coupling is usually implemented so that the magnetic field is first solved by the FE program and the magnetic forces are then calculated. Afterwards, the magnetic forces are used to perform elastic or mechanical analysis to solve the displacement and vibration. Meanwhile, the strong coupling is also an indirect method of determining the effect of the elastic field on the magnetic one and vice versa. This kind of analysis is necessary if the mechanical stress in the machine is too high such that it changes the magnetic properties of the core material [4].

In this study, the strong magneto-mechanical coupling

\footnotetext{
$\dagger$ Corresponding Author: Department of Electrical Engineering, Hongik University, Korea (psshin@hongik.ac.kr)

Department of Electrical Engineering, Hongik University, Korea.

Received: May 25, 2010; Accepted: November 4, 2010
}

formulation is used for the magnetically forced vibration analysis of the large-scale BLDC motor. In the first stage, the initial FE calculation is performed for the large-scale BLDC motor to calculate the nonlinear magnetic field, and the elastic field is then calculated by using the results obtained. Finally, the displacement and vibration modes are evaluated by the FE program. Fig. 1 shows the flow chart of the strong coupling of the magnetoelastic analysis.

To verify the proposed algorithm, $3 \mathrm{MW}$ BLDC motor (32 poles, 192 slots) is simulated [5]. The one-pole pitch model (1/32 model) is used for the magnetic field and the surface force density calculation. The whole model of the stator with frame is used for the calculation of the mechanical vibrations.

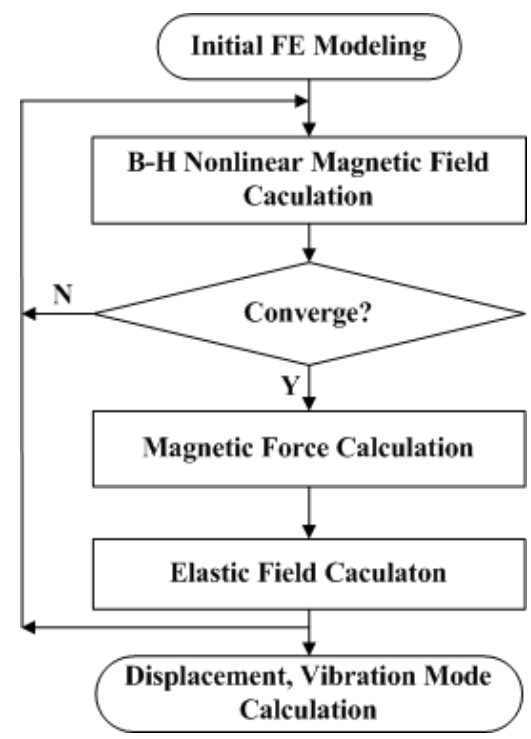

Fig. 1. Flow chart of the strong coupling formulation. 


\section{Coupling Formulation and Mode Calculation}

In the finite element analysis, the strong magnetomechanical coupling formulation is performed by assembling different system matrices for the magnetic and elastic fields. In the program, the magnetic permeability is represented by a set of stress parameterized magnetization curves, whereas the Poisson ratio and Young modulus do not depend on the magnetic field. By using this method, the geometrical changes and magnetostriction can be considered for analysis.

\subsection{Strong Coupling Formulation for Magnetostric- tion}

The electromagnetic force generated by the interaction of load current with the magnet flux is calculated by the Maxwell stress method based on the finite element analysis of the equation, as shown below.

$$
\nabla \times(1 / \mu) \nabla \times A+j \omega_{e} \sigma A=I
$$

Where $\boldsymbol{A}$ is the magnetic vector potential; $\sigma$ is the electrical conductivity; $\omega_{e}$ is the power frequency; $\mu$ is the permeability; and $I$ is the current density. The magnetic flux density, $\boldsymbol{B}$, and the magnetic field intensity, $\boldsymbol{H}$, are estimated by using $\boldsymbol{A}$.

Using the virtual work method, the total magnetic force acting on any part of the machine is calculated from the magnetic energy $\boldsymbol{W}$ as follows:

$$
F^{T}=-\frac{\partial W}{\partial u}=-\frac{\partial}{\partial u} \int\left(\int_{\Omega}^{B} H \cdot d B\right) \operatorname{det}(J) d \Omega
$$

Where the magnetic energy $\boldsymbol{W}$ is described as follows:

$$
W=\int_{\Omega}\left(\int_{0}^{B} H \cdot d B\right) d \Omega
$$

In addition, $\boldsymbol{H}$ and $\boldsymbol{B}$ are the magnetic field intensity and magnetic flux density, respectively; $\boldsymbol{J}$ is the Jacobian matrix of the coordinate transformation; and $\boldsymbol{u}=\left[\mathrm{u}_{\mathrm{t}}, \mathrm{v}_{\mathrm{t}}\right]^{\mathrm{T}}$ is the vector of the virtual displacement. The nodal forces are as follows:

$$
\begin{aligned}
& F^{T}=-\int_{\Omega}\left\{\frac{1}{2} v \frac{\partial B^{2}}{\partial u} \operatorname{det}(J)+\frac{\partial \operatorname{det}(J)}{\partial u} \int_{0}^{B^{2}} \frac{1}{2} v d B^{2}+\right. \\
& \frac{\partial \sigma_{p}}{\partial u} \operatorname{det}(J) \int_{0}^{B^{2}} \frac{1}{2} \frac{\partial v}{\partial \sigma_{p}} d B^{2}+\frac{\partial \sigma_{n}}{\partial u} \operatorname{det}(J) \int_{0}^{B^{2}} \frac{1}{2} \frac{\partial v}{\partial \sigma_{n}} d B^{2}+ \\
& \left.\frac{\partial \sigma_{n}}{\partial u} \operatorname{det}(J) \int_{0}^{B^{2}} \frac{1}{2} \frac{\partial v}{\partial \sigma_{n}} d B^{2}+\frac{\partial \tau}{\partial u} \operatorname{det}(J) \int_{0}^{B^{2}} \frac{1}{2} \frac{\partial v}{\partial \tau} d B^{2}\right\} d \Omega
\end{aligned}
$$

Where $\sigma_{p}, \sigma_{n}$, and $\tau$ are the parallel, orthogonal components to the direction B and shear stress. In the program, for the calculation of nodal forces, the reluctivity of the material is described by the $\mathrm{m}$-dimensional cubic spline approximation with respect to the square of the flux density $\mathrm{B}^{2}$ and the components of the mechanical stress $[\sigma]$. For simplicity, only the component of stress parallel to the flux density is considered [4]. In the field of the $\left[B^{2}, \sigma\right]$ surface, an interval, $\left[B_{k}^{2}, B_{k+1}^{2}\right] \times\left[\sigma_{h}, \sigma_{h+1}\right]$, with respect to the reluctivity $v$ is given by the following:

$$
v\left(B^{2}, \sigma\right)=\sum_{i, j=0,3} a_{k h i j}\left(B^{2}-B_{k}^{2}\right)^{i}\left(\sigma-\sigma_{h}\right)^{j}
$$

The integration and differentiation of the reluctivity with respect to the variables $B^{2}$ or $\sigma$ is carried out analytically using (5). The equation $\frac{\partial B^{2}}{\partial u}$ is calculated by using the derivative of the Jacobian determinant with respect to $\boldsymbol{u}$ and the magnetic vector potential A. Similarly, $\frac{\partial \sigma}{\partial u_{i}}, \frac{\partial \sigma}{\partial v_{i}}$, $\frac{\partial \tau}{\partial u_{i}}$, and $\frac{\partial \tau}{\partial v_{i}}$ can be calculated. An example is shown below:

$$
\frac{\partial\{\sigma\}}{\partial u_{i}}=[E]\left\{\begin{array}{c}
y_{m}-y_{n} \\
v_{m}-v_{n} \\
x_{n}-x_{m}+u_{m}-u_{n}
\end{array}\right\}
$$

Due to the magnetostriction of iron core, an element of the core will shrink or stretch depending on the sign of its magnetostrictive force. This change is described by a magnetostrictive strain tensor $\left\{\varepsilon_{m s}\right\}$, and the corresponding stress tensor $\left\{\sigma_{m s}\right\}$ is calculated using Hook's law. If $\sigma_{m s}$ is the magnetostrictive stress in a direction orthogonal to the magnetic field, then $\sigma_{m s_{-} n}=\frac{2 v-1}{2-v} \varepsilon_{m s_{-} p}$.

The magnetostrictive forces in the original Cartesian coordinate system are evaluated as the projection of $\left\{f_{m s_{-} p}=\sigma_{m s_{-} p} s_{-p}\right\}$ and $\left\{f_{m s_{-} o}\right\}$, which represent the forces of per unit length, parallel $\left(_{-} p\right)$, and orthogonal ( $\_$o ) to the direction of the magnetic field.

$$
\begin{aligned}
& F_{m s x}=\cos (\theta) f_{m s_{-} p}-\sin (\theta) f_{m s_{-} o} \\
& F_{m s y}=\sin (\theta) f_{m s_{-} p}+\cos (\theta) f_{m s_{-} o}
\end{aligned}
$$

These forces are added to the magnetic nodal forces to get the nodal magnetic and magnetostrictive forces. 


\subsection{Vibration Mode Calculation}

After the magnetostrictive forces are calculated by the strong coupling formulation, the whole model is analyzed for displacements and vibration modes. The FE calculation of the dynamic displacements is based on the principle of Hamilton, which prescribes to find the minimum of the difference from the kinetic energy and the elastic potential of the whole structure [6], [7]. Using this principle, the following system of equations is obtained.

$$
\begin{aligned}
M \cdot D^{\prime \prime} & +K \cdot D=F \text { or } \\
& \left(K-\omega_{\text {mech }}^{2} \cdot M\right) \cdot D=F
\end{aligned}
$$

Where $D^{\prime \prime}$ is the global vector of node displacements; $\boldsymbol{M}$ is the global mass matrix including the information of inertia; and $\boldsymbol{K}$ is the global stiffness matrix describing the elastic features of the structure [8]. Meanwhile, $\omega_{\text {mech }}$ is the angular velocity of the stator. The vector $\boldsymbol{F}$ includes the amplitudes of the exciting force densities, which is calculated by the first step of the magnetostrictve coupling analysis. For transient characteristics, a dynamic response analysis is performed at a point by using the mode superposition method [9], [10].

Fig. 2 shows the magnetization curves as functions of externally applied mechanical stress. When the stress is increased, the magnetic flux density is also increased, as shown in the figure. In this study, in order to analyze the effect of the magnetoelastic stress, two magnetization curves are selected: type A is a conventional curve (without mechanical stress) and type B is a curve applied the external magneto-mechanical stress. Type $B$ is under the maximum stress condition with flux density about 5\% higher than that of type A. For a linear approximation of the ferromagnetic material, an expression of the local surface force density, $\kappa$, is given by the Maxwell's stress tensor [7].

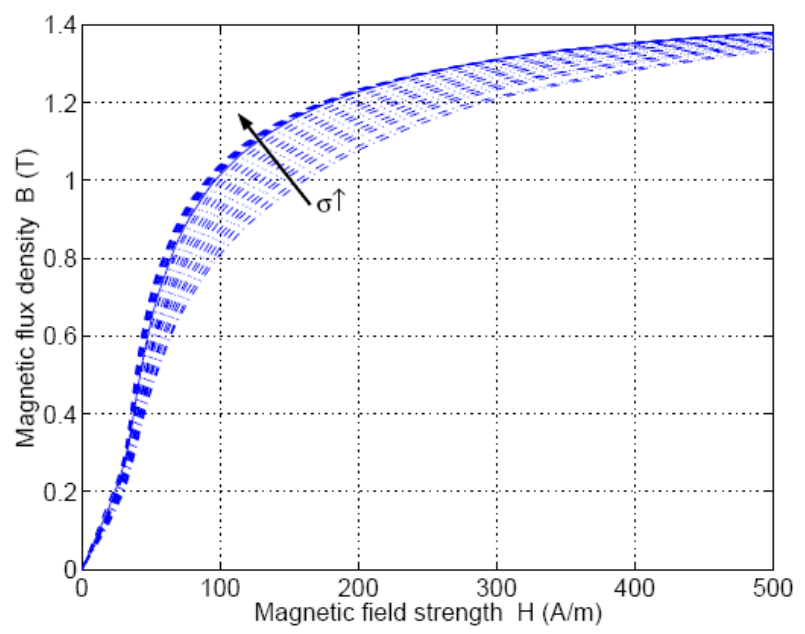

Fig. 2. Magnetization curves as functions of externally applied mechanical stress.

$$
\kappa=\frac{1}{2} n_{12}\left[B_{n}\left(H_{1 n}-H_{2 n}\right)-H_{t}\left(B_{1 t}-B_{2 t}\right)\right]
$$

The values $\boldsymbol{B}$ and $\boldsymbol{H}$ represent the modified magnetic flux density and magnetic field intensity, respectively, and the subscripts $n, 1$, and 2 describe the normal direction, region 1 , and region 2 , respectively (see Fig. 3 ). Some assumptions of the definition of the stress tensor and the electrodynamic field conditions on the boundary (Div $\boldsymbol{B}=$ 0 and Rot $\boldsymbol{H}=0$ ) are shown in (10). Because the force is a periodic function in one pole, the surface force density function must be approximated by a number of models, each with another rotor position. The magnetic force $\boldsymbol{F}$ acting on the stator surface can be calculated by integrating $\kappa$ over a closed surface.

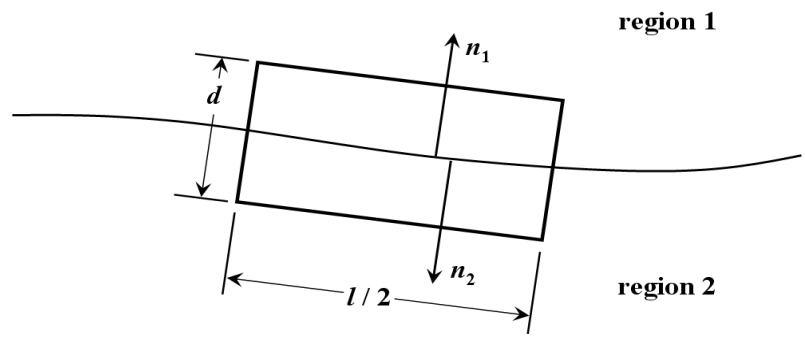

Fig. 3. Boundary condition of the surface force density.

\subsection{Simulation Model and Results}

The FE simulation model of the 3 MW BLDC motor for the electromagnetic force calculation is shown in Fig. 4. Only $1 / 32$ section of the motor is shown in the figure, and its specification is described in Table 1 . The motor has 32 poles, 192 stator slots, 12 phases, and 12 inverter module drivers [11].

The mechanical simulation model for the stress and displacement calculation is shown in Fig. 5. The finite element meshed model with motor frame has about 280,000 nodes and 185,000 tetrahedral meshes.

In the first step of the magneto-mechanical analysis, the airgap flux density is calculated and the force density is derived using (3). When the cogging torque of the model is estimated, there is no difference between the two types of B-H curves. Fig. 6 shows the magnitude of the magnetic force density of one slot pitch in the radial direction. The magnitude of the magnetic force density for type B, which is considered the magneto-mechanical stress, is about $5 \%$ more than those of type A. Fig. 7(a) shows the vector diagram of the magnetic force density for the whole model, while Fig. 7(b) shows the enlarged vector diagram with the color-shaded magnetic flux density of the two poles. The electromagnetic force vectors have periodic pattern every six slots, as expected. Figs. 8(a), 8(b), 8(c), and 8(d) show the forced vibration modes of the $3 \mathrm{MW}$ BLDC motor with stator frame; the frequencies of the modes are 60.5, 84.2, 127.6, and $199.2 \mathrm{~Hz}$, respectively. The displacements of the modes are magnified by 900 times to distinguish the forced vibration modes. 
From the results of the displacement calculation, the maximum deformation (for the conventional B-H curve type A) is $0.125 \mathrm{~mm}$ and the minimum $0.101 \mathrm{~mm}$. The type B model is about $5 \%$ more deformed than type A. Fig. 9 shows a time response of the displacement on a point of the stator to the radial direction. The maximum displacement is $0.104 \mathrm{~mm}$ and the minimum is $0.025 \mathrm{~mm}$.

Table 1. Specification of the 3 MW BLDC motor

\begin{tabular}{c|c|c}
\hline Parameters & Value & Unit \\
\hline Maximum output & 3,000 & $\mathrm{~kW}$ \\
\hline Rated voltage/current & $650 / 450$ & $\mathrm{~V} / \mathrm{A}$ \\
\hline Rated speed & 150 & $\mathrm{rpm}$ \\
\hline Number of phase & 12 & $\mathrm{phase}$ \\
\hline Outer diameter(stator/rotor) & $1,700 / 1500$ & $\mathrm{~mm}$ \\
\hline Air gap length & 10.0 & $\mathrm{~mm}$ \\
\hline Stator core length & 850 & $\mathrm{~mm}$ \\
\hline
\end{tabular}

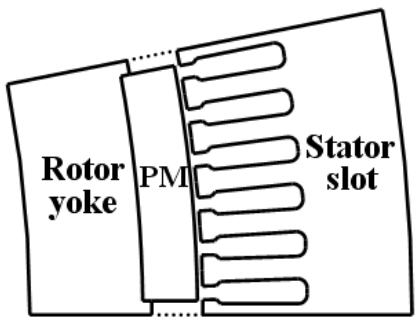

Fig. 4. FEM model of the 3 MW BLDC motor (1/32 model).

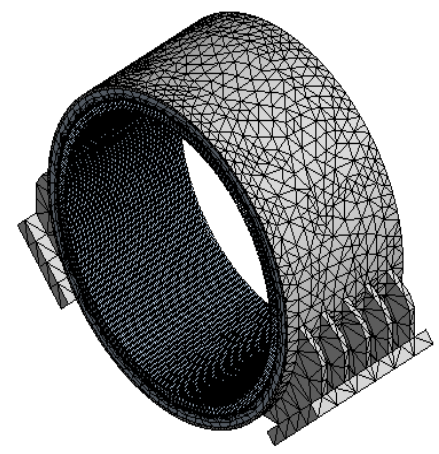

Fig. 5. Vibration analysis model of the stator with frame.

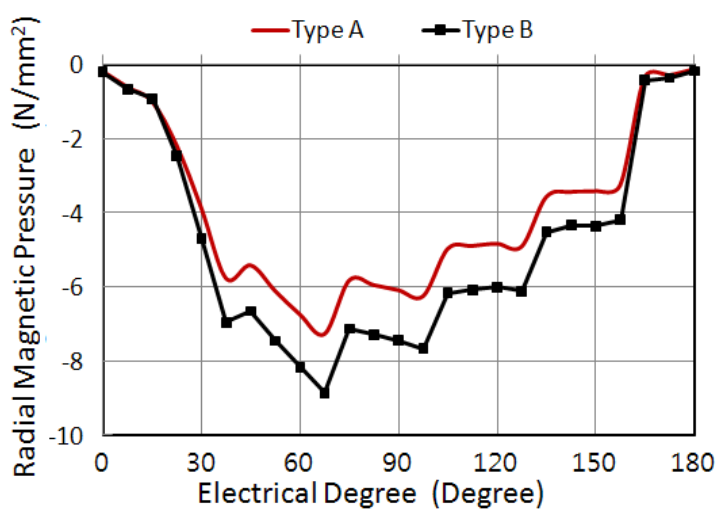

Fig. 6. Magnetic force density of one slot pitch in the radial direction.

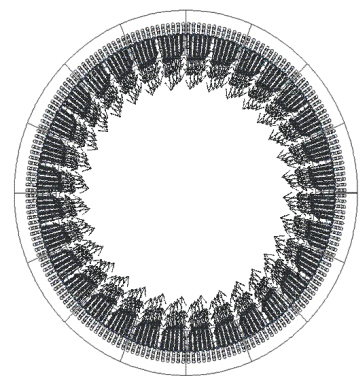

(a) Vector diagram

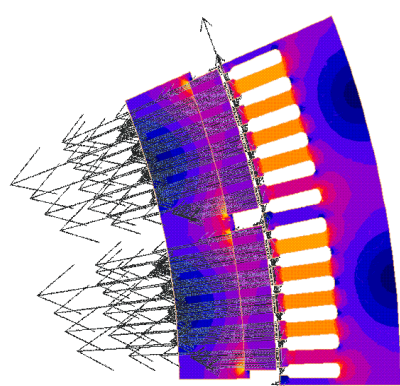

(b) Enlarged vector diagram (two poles)
Fig. 7. Electromagnetic force density vector diagram on the stator.

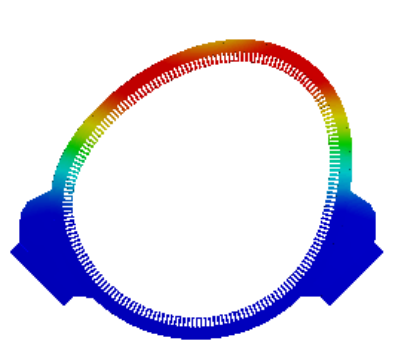

(a) First mode $(60.5 \mathrm{~Hz})$

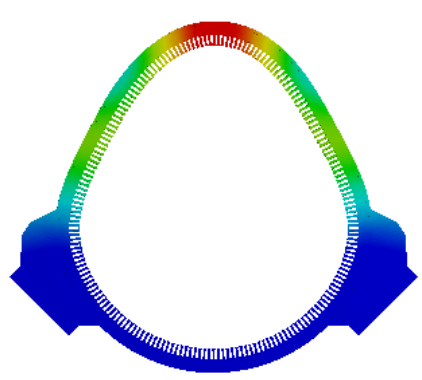

(c) Third mode $(127.6 \mathrm{~Hz})$

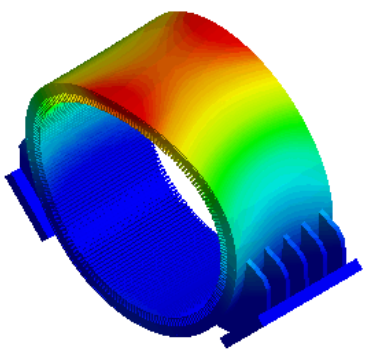

(b) Second mode $(84.2 \mathrm{~Hz})$

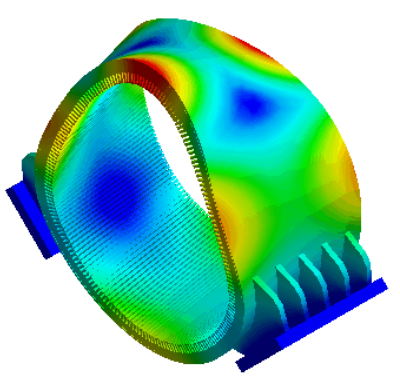

(d) Fifth mode $(199.2 \mathrm{~Hz})$
Fig. 8. Forced vibration modes of the BLDC motor with the stator frame $(900 \times$ magnification $)$.

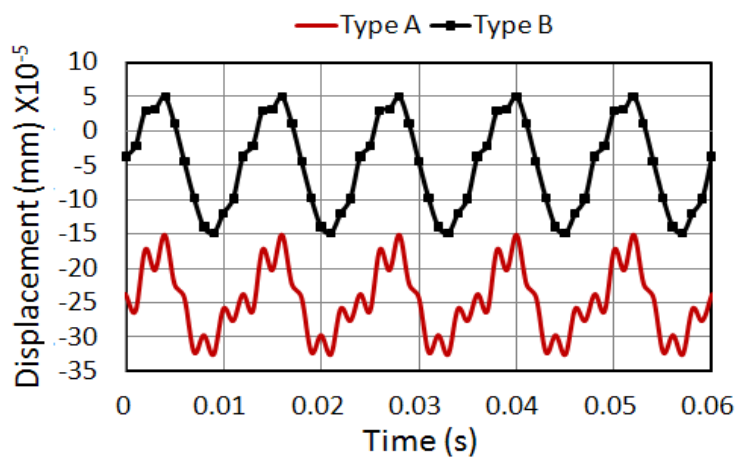

Fig. 9. A time response of displacement on a point of the stator (radial direction).

\section{Conclusion}

A magneto-mechanical coupling method was proposed to calculate the magnetoelastic forces, displacement, and 
vibration modes of the large-scale BLDC motor by using the magneto-mechanical finite element coupling formulation. The force on the core was calculated by the energy method coupled with magnetostrictive stress tensor. The mechanical vibration modes were also analyzed by using the principle of Hamilton and the calculated magnetoelastic forces acting on the surfaces of the stator.

In addition, the externally applied mechanical stress of the core was considered for estimating the effects of the magnetostriction. To verify the algorithm, the $3 \mathrm{MW}$ BLDC motor was simulated, and the air gap flux density, forces and displacement and the vibration modes were analyzed. The results show that the mechanically stressed core has more deformation or displacements than the normal by about $5 \%$.

The method suggested in the paper would be a noble technique to analyze a magnetostriction problem as well as magneto-mechanical, if the computing procedure would be improved with a strong coupling algorithm.

\section{Acknowledgements}

This work was supported by the research grant from KEPEP (R-2008-32) of the Ministry of Knowledge and Economy, Korea in 2010.

\section{References}

[1] Z. Ren, A. Razek, "Local Force Computation in Deformable Bodies Using Edge Elements", IEEE Trans on Magnetics, Vol. 28. No. 2, March 1992, pp. 12121215.

[2] O. Mohammed, T.E. Calvert, and R. McConnell, "A Model for Magnetostriction in Coupled Nonlinear Finite Element Magneto-Elastic Problems in Electrical Machines", Proceedings of the International Conference on Electric Machines and Drives, pp. 728-735, 1999.

[3] O. Mohammed, T.E. Calvert, and R. McConnell, "Transient Modeling of Coupled Magnetoelastic Problems in Electrical Machines", Proceedings of the Power Engineering Society Summer Meeting, Vol. 1, pp. 281-287, July 2002.

[4] A. Belahcen, "Magnetoelasticity, Magnetic Forces and Magnetostriction in Electrical Machines", Doctoral thesis, Helsinki University of Technology, August 2004.
[5] P.S. Shin, H,K. Chung, S.H Woo, "A Magnet Pole Shape Optimization of a Large Scale BLDC Motor Using a RSM with Design Sensitivity Analysis", Journal of KIEE, Vol. 58, No. 4, pp. 735-742, April 2009.

[6] R.D. Cook, 'Concepts and applications of finite element analysis', New York Wiley 1974.

[7] G. Henneberger, and et.al., "Procedure for the numerical computation of Mechanical Vibrations in Electrical machines", IEEE Trans on Magnetics, Vol. 28, No. 2, pp. 1351-1354, March 1992.

[8] I. Ramesohl, and et.al., "Three Dimensional Calculation of Magnetic Forces and Displacements of a Claw-Pole Generator", IEEE Trans on Magnetics, Vol. 32, No. 3, pp. 1685-1688, March 1992.

[9] H.J. Cheong, P.S. Shin and S.H. Woo, "Vibration Analysis for BLDC Motor by Electromagnetic Exciting Force", Proceeding of KIEE Annual Meeting(B), October 2007.

[10] Hee Jun Cheong, "A Vibration Analysis for Large Scale BLDC motor by Electromagnetic Exciting Force", Master Thesis (in Korean), Hongik University, Korea, August 2008.

[11] S.H.Woo, P.S.Shin, J.S.Oh, Y.K.Kong and J.G. Bin, "A Study on the Optimization Strategy using Permanent Magnet Pole Shape Optimization of a Large Scale BLDC Motor', Journal of KIEE, Vol. 59, No. 5, pp. 897-903, May 2010.

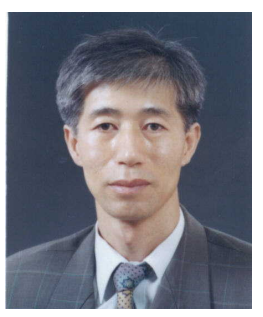

Pan Seok Shin received his B.S. degree in Electrical Engineering from Seoul National University and his M.S. and Ph.D. degrees from Rensselaer Polytechnic Institute (USA). He works at the Electrical Engineering department of Hongik University. His research interests are electromagnetic field analysis and electric machinery design and simulations

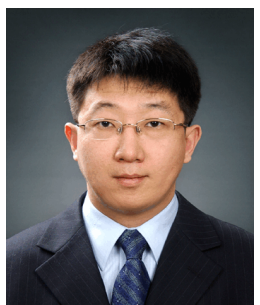

Hee Jun Chung received his B.S. degree in Mechanical Engineering from Dongguk University and his MS degree from Hongik University. $\mathrm{He}$ works at Gausstech, Inc. His research interests are mechanical application of electromagnetic field analysis and simulations. 